\title{
AVALIAÇÃO DA EFETIVIDADE DO "VAMOS" NO CONTEXTO DO PROGRAMA ACADEMIA DA SAÚDE: UM ESTUDO QUALITATIVO
}

\author{
EVALUATING THE EFFECTIVENESS OF PROGRAM VAMOS IN THE \\ CONTEXT OF PROGRAM ACADEMY OF HEALTH: A QUALITATIVE \\ STUDY
}

\section{EVALUACIÓN DE LA EFECTIVIDAD DEL "VAMOS" EN EL CONTEXTO DEL PROGRAMA ACADEMIA DE LA SALUD: UN ESTUDIO \\ CUALITATIVO}

\author{
Emanuele Naiara Quadros*, Elaine Cristina Maciel*, \\ Lisandra Maria Konrad*, Cezar Grontowski Ribeiro*, \\ Aline Cristine Souza Lopes ${ }^{* *}$, Simone Teresinha Meurer ${ }^{* * *}$, \\ Tânia Rosane Bertoldo Benedetti*
}

Palavras chave: Atividade Motora Alimentação Saudável. Mudança de Comportamento. Promoção da Saúde.
Keywords: Physical Activity. Healthy Eating. Behavior Change. Health Promotion.

Palabras clave: Actividad Motora Alimentación Saludable. Cambio de Comportamiento. Promoción de la Salud.
Resumo: O objetivo deste estudo foi avaliar a efetividade do Vida Ativa Melhorando a Saúde (VAMOS) a partir da percepção dos usuários do Programa Academia da Saúde (PAS) de Belo Horizonte. Foi realizado um estudo qualitativo com participantes do GI (Grupo Intervenção) de um ensaio controlado e randomizado em dois polos do PAS. O GI participou das atividades do PAS e, adicionalmente, de um programa de mudança de comportamento (VAMOS) durante 12 semanas. A efetividade foi avaliada por meio de relatos em três grupos focais realizados com 32 participantes do $\mathrm{GI}(61$ anos, $\pm 7,07)$ utilizando o roteiro baseado nas dimensões do modelo RE-AIM. A partir da análise de conteúdo foram identificados benefícios em quatro categorias: atividade física, alimentação, peso corporal e qualidade de vida. Pela percepção dos participantes, concluiu-se que o VAMOS trouxe benefícios nos marcadores do programa e que a metodologia utilizada foi efetiva para atingir os resultados.

Abstract: This study evaluates the effectiveness of program VAMOS (Active Life Improving Health) based on the perception of the Health Academy Program (PAS) users in Belo Horizonte. A qualitative study was conducted with participants of a randomized clinical trial in two PAS units. The IG (Intervention Group) participated in PAS activities and a behavioral change program (VAMOS) for 12 weeks. Effectiveness was evaluated through reports on three focus groups with 32 IG participants (aged $61 \pm 7.07$ ) using the script based on the dimensions of the RE-AIM framework. Through content analysis, benefits were identified in four categories: physical activity, food intake, body weight, and quality of life. Participants' perception reflected benefits in the program markers. Therefore, the methodology used proved to be effective in achieving the VAMOS goals.

Resumen: El objetivo de este estudio fue evaluar la efectividad del "Vida Activa Mejorando la Salud" (VAMOS) desde la percepción de los usuarios del Programa Academia de la Salud (PAS) de Belo Horizonte. El estudio fue cualitativo, con participantes del GI (Grupo Intervención) en un ensayo controlado aleatorio en dos polos del PAS. El GI participó en las actividades del PAS y en un programa de cambio de comportamiento (VAMOS) durante 12 semanas. La efectividad se evaluó a través de relatos en tres grupos focales realizados con 32 participantes del GI $(61$ años, $\pm 7,07)$ utilizando el guión basado en el modelo RE-AIM. A partir del análisis de contenido, se identificaron beneficios en cuatro categorías: actividad física, alimentación, peso corporal y calidad de vida. A partir de la percepción de los participantes, se concluyó que el VAMOS trajo beneficios en los marcadores del programa y que la metodología utilizada fue efectiva para alcanzar los resultados.
*Universidade Federal de Santa Catarina. Florianópolis, SC, Brasil. E-mail:

nueleq@gmail.com;

elainecristinamaciel86@gmail.com; lisandrakonrad@hotmail.com; cezar.ribeiro@ifpr.edu.br; tania.benedetti@ufsc.br

**Universidade Federal de Minas Gerais. Belo Horizonte, MG, Brasil. E-mail:

alinelopesenf@gmail.com

***Instituto Federal de Minas Gerais Belo Horizonte, MG, Brasil. E-mail: simonemeurer@yahoo.com.br

Recebido em: 11-07-2019 Aprovado em: 12-03-2020 Publicado em: 29-03-2020 


\section{INTRODUÇÃO}

Baseada no cuidado integral, articulada e territorializada, a Atenção Básica a Saúde exerce papel fundamental no atual modelo de atenção à saúde. Por estar inserida próxima à comunidade propicia a criação de vínculos com usuários e tem maior poder de compreensão da dinâmica social que impacta a vida e a saúde da população (BRASIL, 2017). A Atenção Básica é um espaço privilegiado para o desenvolvimento de ações de promoção da saúde.

Atualmente, $47 \%$ dos programas comunitários de promoção da atividade física (AF) e $72 \%$ das ações de alimentação saudável (AS) são realizados na Atenção Básica (BECKER; GONÇALVES; REIS, 2016). O cenário nacional, certamente é influenciado pela expansão do Programa Academia da Saúde (PAS) (SÁ et al., 2016).

OPAS se destaca como um programa que visa qualificar as ações de promoção da saúde. Preconiza-se que, as atividades desenvolvidas em seu âmbito sejam culturalmente inseridas e adaptadas aos territórios, sendo um potencial elemento para o aumento dos níveis de AF na população (MACIEL et al., 2019; MALTA et al., 2014; BRASIL, 2013). De acordo com o sistema de monitoramento do PAS, em 2017, o número de polos habilitados era de 4.271 em todas as regiões brasileiras e suas atividades alcançaram mais de 2.500 municípios, com potencial para cobrir um milhão de pessoas (FLORINDO et al., 2016).

Estudo realizado com polos em funcionamento no país revelou que $96 \%$ oferecem AF, 94\% atividades de educação em saúde e 91\% ações de AS (SÁ et al., 2016). As ações e estratégias desenvolvidas no PAS são as mais diversas, contudo, programas de mudança de comportamento também têm se mostrado promissores. Estas intervenções não necessitam de equipamentos específicos, podem ser conduzidas por diferentes profissionais da saúde e associadas a promoção de outros comportamentos (RIBEIRO et al., 2017). Dos programas de mudança de comportamento comunitários, $53,8 \%$ integram a promoção da AF com a AS (KONRAD et al., 2018).

Nessa perspectiva, novas propostas de intervenções têm sido testadas, dentre elas o Vida Ativa Melhorando a Saúde (VAMOS) que é um programa de mudança de comportamento, embasado na teoria sociocognitiva (BANDURA, 1986). Seu objetivo é motivar as pessoas a adotarem um estilo de vida ativo e saudável com relação à $A F$ e a alimentação (BENEDETTI et al., 2017; BENEDETTI et al., 2012). Um diferencial do programa é a utilização do modelo RE-AIM, desenvolvido para avaliar e planejar o impacto de programas na saúde pública por meio de cinco dimensões: alcance, adoção, efetividade, implementação e manutenção (GLASGOW; VOGT; BOLES, 1999). Esse modelo visa contribuir, tanto em nível individual quanto organizacional, no planejamento, na avaliação e no monitoramento de intervenções (ALMEIDA; BRITO; ESTABROOKS, 2013).

No Brasil, ainda é incipiente o que se sabe sobre a efetividade das intervenções com programas de mudança de comportamento (CAMPOS, 2017; FLORINDO et al., 2014). O VAMOS tem-se mostrado efetivo em relação aos principais desfechos (AF e AS). Ensaios controlados e randomizados mostraram 
redução do comportamento sedentário, aumento da AF leve e incremento de $10 \%$ no tempo de AF em intensidades moderada/vigorosa (GERAGE et al., 2017), menor consumo de temperos industrializados, aumento do consumo de verduras e legumes (MEURER et al., 2019) e melhora na percepção da qualidade de vida (QV). Alguns resultados apontam que a metodologia empregada foi um facilitador para a mudança nos comportamentos (TONOSAKI et al., 2018).

Contudo, além das avaliações com instrumentos objetivos e análises quantitativas, a compreensão de como os participantes percebem os benefícios é essencial para potencializar as intervenções. Portanto, o objetivo deste estudo foi analisar a efetividade do VAMOS a partir da percepção dos usuários do Programa Academia da Saúde de Belo Horizonte, MG.

\section{METODOLOGIA}

Este estudo é recorte de um ensaio controlado e randomizado conduzido em dois polos do PAS na cidade Belo Horizonte no ano de 2015 (MEURER, 2016). Participaram do grupo intervenção (GI) 135 usuários, distribuídas em oito turmas com até 20 participantes. O GI realizou as atividades rotineiras do PAS (exercícios físicos, três vezes na semana, 60 minutos por dia) e, adicionalmente, participou do VAMOS (um encontro semanal com duração de 60 minutos) no decorrer de 12 semanas consecutivas. Em cada encontro foi desenvolvida uma temática específica (Quadro 1) mediada por um profissional previamente treinado. Detalhamento metodológico do estudo está publicado em Meurer et al. (2019).

Quadro 1 - Temas abordados no Programa Vida Ativa Melhorando a Saúde (VAMOS), 2017.

\begin{tabular}{|l|}
\hline Encontro 1. Introdução \\
\hline Encontro 2. VAMOS preparar? \\
\hline Encontro 3. VAMOS saber mais sobre alimentação saudável? \\
\hline Encontro 4. VAMOS decidir e praticar? \\
\hline Encontro 5. VAMOS superar desafios? \\
\hline Encontro 6. VAMOS estabelecer metas? \\
\hline Encontro 7. VAMOS reunir apoio e ir passo a passo? \\
\hline Encontro 8. VAMOS ganhar confiança e revisar os objetivos? \\
\hline Encontro 9. VAMOS enfrentar os obstáculos? \\
\hline Encontro 10. VAMOS desfazer o estresse? \\
\hline Encontro 11. VAMOS encontrar novas oportunidades para ter uma vida saudável? \\
\hline Encontro 12. VAMOS realizar mudanças duradouras? \\
\hline
\end{tabular}

Fonte: Adaptado de Benedetti et al. (2017).

Para este estudo foram conduzidos três Grupos Focais (GFs), com até 11 participantes cada. Os participantes do primeiro e segundo grupos foram selecionados aleatoriamente (sorteio) usando como critério a frequência mínima de $75 \%$ nos encontros do VAMOS e convidados verbalmente para participar do GF. Para o terceiro GF foram convidados dez participantes considerados informantes-chaves 
pelo mediador dos encontros do VAMOS. A concepção de informante-chave adotada nesse estudo baseou-se nas ideias formuladas por Minayo que os considera como informantes pessoas estratégicas que podem representar o grupo (MINAYO, 1992).

Os GFs foram conduzidos por uma profissional treinada (nutricionista e pesquisadora na área de nutrição) e que não teve qualquer contato prévio com os participantes. No dia, hora e local pré-agendados no PAS, a profissional se apresentou e iniciou o GF, tendo em média 56 minutos de duração. Os relatos foram gravados em áudio e transcritos na íntegra. $O$ roteiro de perguntas seguiu os itens das dimensões do modelo RE-AIM (Adoção, Alcance, Implementação, Efetividade/ Eficácia e Manutenção). As perguntas norteadoras para os GFs analisadas neste estudo foram: "Ao participar do VAMOS, vocês perceberam alguma mudança? Contem-nos o que vocês perceberam? Como o Programa VAMOS contribuiu para a vida de vocês?".

Para o tratamento dos dados foi utilizado o software NVIVO 12.0 e optouse pela análise de conteúdo (BARDIN, 2009). Realizada de maneira sistemática, a análise de conteúdo é composta por quatro fases: pré-análise, exploração do material, tratamento dos resultados e interpretação, possibilitando a categorização, descrição e interpretação dos dados. Na pré-análise foi realizado um contato prévio com os documentos a fim de definir categorias para a exploração do material. Na exploração do material foram realizadas leituras, definindo os objetivos e a caracterização da amostra. Para o tratamento dos resultados foi realizada a análise do conteúdo e a categorização dos temas pertinentes ao estudo. Na fase da interpretação foram analisados os relatos presentes em cada categoria de forma qualitativa referente à percepção da efetividade pelos participantes.

Três pesquisadores realizaram a categorização das informações, análise do conteúdo e escolha dos relatos, sendo o terceiro necessário para eventuais divergências. Para manter o anonimato dos participantes dos GFs, optou-se por identificá-los no texto por meio de combinação de letras e números (P1 .... P32). Os dados de caracterização da amostra foram apresentados por meio de frequência absoluta e relativa, valores médios e percentuais. $O$ estudo foi estruturado seguindo o checklist do Consolidated Criteria for Reportin Qualitative Research (COREQ) (TONG; SAINSBURY; CRAIG, 2007).

O estudo foi aprovado pelo Comitê de Ética em Pesquisa com Seres Humanos da Secretaria Estadual da Saúde do Estado de Santa Catarina (parecer n 767.704) e registrado no clinicaltrials.gov, sob n NCT02659267.

\section{RESULTADOS}

Na tabela 1 são apresentados os dados sociodemográficos dos participantes do VAMOS e dos GFs. Os participantes eram predominantemente do sexo feminino, com média etária acima de 60 anos, aposentados e com cerca de 10 anos de estudos. Verificou-se que os participantes do GF tinham perfis similares aos participantes do programa. 
Tabela 1 - Características sociodemográficas dos participantes do VAMOS, Belo Horizonte, Brasil, 2019.

\begin{tabular}{|c|c|c|}
\hline Variáveis & $\begin{array}{l}\text { Participantes do VAMOS } \\
\qquad(\mathrm{N}=135)\end{array}$ & $\begin{array}{c}\text { Participantes dos Grupos } \\
\text { Focais }(\mathrm{N}=32)\end{array}$ \\
\hline $\begin{array}{l}\text { Sexo } \\
\qquad \text { Mulheres, } n(\%)\end{array}$ & $125(92,6)$ & $31(96,9)$ \\
\hline $\begin{array}{l}\text { Idade (anos) } \\
\text { Mín - Máx } \\
\text { Média }(D P) \\
\end{array}$ & $\begin{array}{c}30-84 \\
61,5(9,4) \\
\end{array}$ & $\begin{array}{c}41-82 \\
61,1(9,4) \\
\end{array}$ \\
\hline $\begin{array}{l}\text { Estado Civil, } n(\%) \\
\text { Com companheiro } \\
\text { Sem companheiro }\end{array}$ & $\begin{array}{l}68(50,4 \%) \\
67(49,6 \%)\end{array}$ & $\begin{array}{l}18(56,3) \\
14(43,7)\end{array}$ \\
\hline $\begin{array}{l}\text { Ocupação, } n(\%) \\
\text { Aposentado } \\
\text { Do lar } \\
\text { Outros }\end{array}$ & $\begin{array}{l}58(43,0) \\
32(23,7) \\
45(33,3)\end{array}$ & $\begin{array}{l}13(40,6) \\
09(28,1) \\
10(31,3)\end{array}$ \\
\hline $\begin{array}{l}\text { Anos de estudo } \\
\text { Mín - Máx } \\
\text { Média }(D P)\end{array}$ & $\begin{array}{c}0-21 \\
9,2(4,5)\end{array}$ & $\begin{array}{c}2-21 \\
10,5(5,3)\end{array}$ \\
\hline
\end{tabular}

Fonte: Os autores (2019).

A partir da análise dos relatos dos GFs foram identificadas quatro categorias relacionadas à percepção de efetividade do VAMOS: AF, alimentação, peso corporal e QV (Figura 1).

Figura 1 - Categorias dos benefícios percebidos pelos participantes do Programa VAMOS, 2015.

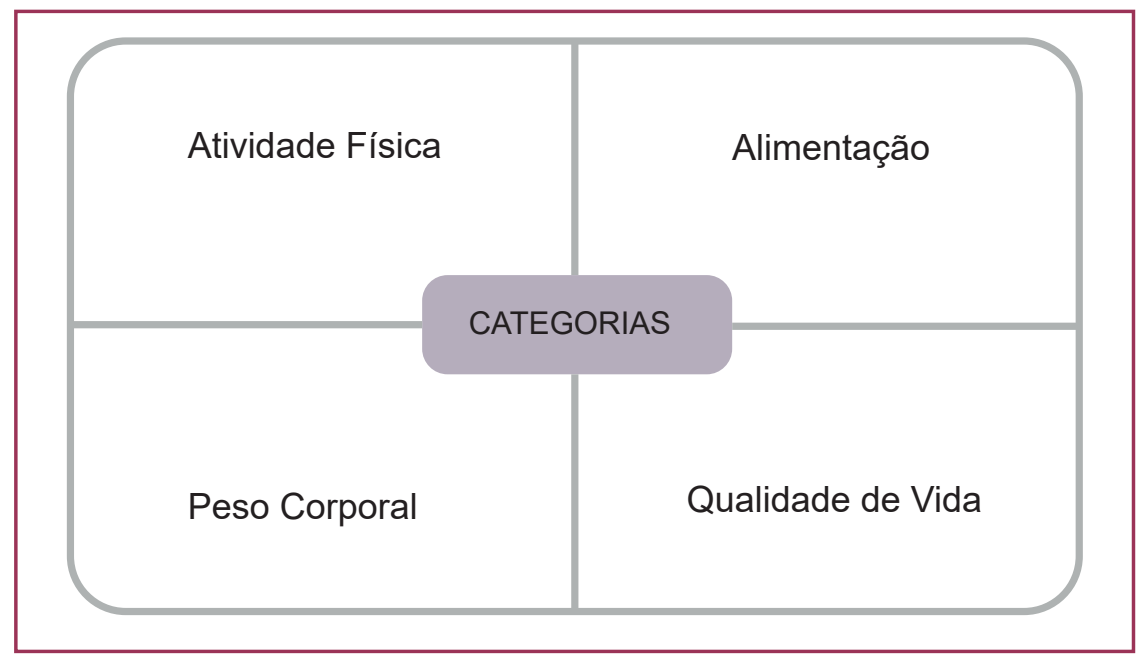

Fonte: Os autores (2019).

\subsection{ATIVIDADE FÍSICA}

Os benefícios percebidos na $A F$ dizem respeito à frequência e ao tipo de atividade inserida na rotina dos participantes da intervenção. Estes benefícios podem ser observados nas seguintes falas:

[...] com relação aos exercícios, a questão de sempre procurar fazer mais um pouco, porque eu tinha sempre aquele ritmo de anos e anos, sempre a mesma coisa, mas sempre gostei de caminhar, de fazer exercício, mas a questão de sempre melhorando o seu nível de exercício, aumentando sabe, isso também foi um despertar pra mim. (P13) 
[...] sabe o que eu to fazendo? Correndo maratona, jamais pensei. Já vou pra terceira maratona agora esse mês correr, andei e corri, porque eu não consigo correr o tempo todo, $5 \mathrm{~km}$. (P5)

[...] Uma coisa que eu achei muito bom também foi aqueles exercícios que tem nesse programa né, ensinando ali as figuras e tudo, a gente pode fazer em casa né, pra alongamento, fortalecimento muscular, eu acho que tudo é importante. (P8)

\subsection{ALIMENTAÇÃO}

Esta categoria foi a que apresentou um maior número de relatos sobre benefícios percebidos entre os participantes do programa, demonstrando mudanças de comportamento sensíveis na vida dos participantes e de seus familiares. Como é possível observar nas falas, o VAMOS proporcionou alterações na maneira de se alimentar, maior conscientização para escolha dos alimentos durante as compras e na interpretação dos rótulos para obter maiores informações sobre os alimentos escolhidos.

[...] essa coisa de mudar as compras, minhas compras elas tinham valor alto e só levava coisa que não era saudável pra dentro de casa, agora a minha lista de compras diminuiu muito mais e eu como muito melhor, muito mais saudável. (P5)

É o programa mudou para mim, achei ótimo, porque eu tinha aquele ritmo de tomar café pela manhã, almoçar e jantar. Então através do programa eu sei que tem que fazer as alimentações intermediárias. (P32)

[...] esclareceu muita coisa, sobre os sucos, o que a gente deve comprar pra usar, sobre os embutidos, sobre o queijo, que o queijo bom é o branco, que não tem gordura, essas coisas assim eu melhorei, eu procurei melhorar minha alimentação e fiquei mais animada pra andar.(P26)

Pra mim o VAMOS foi assim um alerta pra eu me conscientizar do que tem que ser feito na realidade, por exemplo, essa questão do supermercado, chegava à vontade. Aprendi a ter mais atenção com os rótulos, a fazer compras, assim, mais consciente. (P13)

\subsection{PESO CORPORAL}

As mudanças nos comportamentos na prática da AF e hábitos alimentares trouxeram benefícios tanto para quem almejava reduzir ou aumentar o peso.

[...] eu comecei a emagrecer, então assim foi uma coisa muito boa, porque realmente eu preciso emagrecer mesmo, questão de saúde também. (P9)

[...] já comecei também a melhorar também a minha vida. Até que eu já engordei um pouquinho. Eu estava com 38, 35 não dava né [...] (P3)

[...] eu queria manter os $45 \mathrm{~kg}$ que eu tenho que é o meu peso normal, então eu fiquei, eu consegui dentro desse programa, eu consegui. (P7)

\subsection{QUALIDADE DE VIDA}

A percepção de melhora na $\mathrm{QV}$ também foi relatada pelos participantes do estudo, mostrando que aspectos psicológicos (autoestima) e sociais foram impactados pela intervenção.

Eu acho muito importante porque eu mesmo aprendi mais a gostar mais de mim, a valorizar mais sabe dar mais valor, tirar mais tempo pra mim, eu 
não preocupava comigo mesmo sabe, em tirar um tempo e eu acho que nós precisamos de um tempo né pra gente mesmo né, e assim fiquei com mais ânimo. (P6)

Autoestima lá no alto. A minha era assim zerada, naquela última brincadeira de olhar e ver seu rosto no espelho, eu falei assim: há uns seis meses atrás eu queria enterrar essa pessoa. (P29)

[...] o que mais me ajudou na socialização, porque eu era uma pessoa assim muito tímida. (P7)

Além das categorias citadas, foram identificados relatos de que a metodologia utilizada e o papel do profissional - multiplicador - que conduziu os encontros do programa influenciaram na percepção positiva dos participantes sobre as mudanças ocorridas.

[...] o VAMOS foi assim um alerta pra eu me conscientizar do que tem que ser feito na realidade. (P13)

Ter força de vontade né, então eu aprendi muito, aprendi tudo isso aqui no VAMOS, ter força de vontade e manter né para o nosso bem mesmo. (P6)

O diferencial do programa é justamente esse, não é uma dieta, é uma educação que você passa. (P30).

E ela (a multiplicadora) colocou assim de uma forma muita prática, pra gente conscientizar com exemplos, então assim muito positivo pra todos nós. (P21)

Toda vez que eu falo vou perder eu lembro dela (a multiplicadora) que vai me ajudar. (P29)

[...] simplesmente me surpreendi, pela competência dela (multiplicadora), o jeito dela levar as reuniões, supercompetente, super compreensiva." (P17)

\section{DISCUSSÃO}

A partir dos relatos dos participantes foram identificadas quatro categorias de benefícios advindos da participação no VAMOS: melhora na AF, na alimentação, no peso corporal e na QV. É importante relembrar que as categorias que surgiram na análise dos dados dos GFs são preconizadas no VAMOS como desfechos primários (melhora nos níveis de AF e na escolha de alimentos saudáveis) e desfechos secundários (melhora na QV e do peso corporal).

A AF é reconhecida como estratégia efetiva para proteção e tratamento de doenças crônicas não transmissíveis (OLIVEIRA; GORDIA; QUADROS, 2017). Contudo, Carvalho e Nogueira (2016) alertam sobre a necessária compreensão do fenômeno da AF, especialmente no contexto da promoção da saúde na $A B$. Nesse cenário, é fundamental não ter uma visão simplista, de forma que as práticas de promoção da saúde se tornam descontextualizadas. A AF na promoção da saúde deve ser considerada, como parte de ações integradoras e descentralizadas que recebem uma vasta participação social na melhora das condições de vida da população (CARVALHO; NOGUEIRA, 2016).

Os resultados apontam que a intervenção permitiu reflexão, compreensão, maior autonomia e prontidão para a prática de AF entre usuários do PAS. Identificou-se ainda que os participantes do VAMOS tiveram oportunidade de conhecer mais sobre AF, 
ampliar a intensidade das atividades e reconhecer a importância para a saúde. Outro estudo (KNUTH et al., 2011) também observou que indivíduos expostos a programas comunitários de promoção da AF como o CuritibAtiva, Academia da Cidade de Recife e Academia da Cidade de Aracaju, são mais ativos no lazer. Estes resultados demonstram a importância de associar ações de promoção da saúde no contexto do SUS.

As estratégias utilizadas na intervenção do VAMOS buscam aproximação da realidade dos participantes. Como exemplo, foram identificados espaços adequados para a prática de AF próximos à residência dos participantes, ampliando assim, as opções de lazer ativo desses. Considerando a potencialidade das intervenções quando inseridas e articuladas ao território, há necessidade dos programas de promoção da saúde considerarem as condições de vida de seus participantes. Esta compreensão se faz necessária posto que os determinantes sociais (cultural, ambiental, político, econômico) influenciam diretamente no processo de saúde individual e coletiva. Os programas atuais devem valorizar as experiências dos indivíduos em todas as esferas e contextos, e, empenhar-se para atender princípios e valores da promoção da saúde (CARVALHO; NOGUEIRA, 2016; GONÇALVES et al., 2019).

O plano de ação global para a AF (2018-2030) (Let's Be Active) apresenta um conjunto de ferramentas que podem ser facilitadas pelas políticas públicas para aumentar a AF da população. Ele aborda determinantes culturais, ambientais e individuais, por meio de ações de políticas intersetoriais de planejamento urbano buscando criar sociedades, ambientes, pessoas e sistemas mais ativos (WHO, 2018). Nesse sentido, o VAMOS foi estruturado e testado no contexto da atenção básica e, apesar de ter uma estrutura previamente proposta, ele tem na sua metodologia (roda de conversa), a possibilidade de trazer as ações para a realidade dos participantes. O programa fomenta a reflexão sobre as dificuldades individuais e coletivas para a mudança de comportamento - AF e alimentação, consolidando os saberes saudáveis.

Com relação a alimentação, os resultados apontam benefícios da intervenção sobre maior conscientização para a escolha dos alimentos, baseandose nas informações nutricionais dos rótulos. Acredita-se que com a ampliação dos conhecimentos sobre alimentação e nutrição contribuiu para mudanças nesse comportamento. O VAMOS consegue aproximar-se da realidade dos seus participantes, pois discute conceitos relativos à AS a partir do Guia Alimentar para a População Brasileira (BRASIL, 2014; FRAGA et al., 2013; CAVALCANTI; OLIVEIRA, 2012). O estudo de GERAGE et al. (2017), que desenvolveu a intervenção do VAMOS com hipertensos de Recife, também relatou melhora nos hábitos alimentares dos participantes do VAMOS.

A percepção dos benefícios para a saúde foi a principal causa para o aumento de consumo de alimentos saudáveis entre os usuários do PAS, conforme o estudo de Figueira, Lopes e Modena, (2016). Por outro lado, o estudo de Lopes, Menezes e Araújo (2017) identificou que as iniciativas para uma alimentação mais saudável e os estabelecimentos comerciais de frutas e hortaliças da cidade de Belo Horizonte ainda se encontravam concentradas nas regiões consideradas nobres e eram limitadas nas periferias e nas proximidades dos PAS. Já Araújo (2016) verificou que há baixa disponibilidade e a má qualidade de frutas e hortaliças nos territórios do entorno 
dos PAS de Belo Horizonte. Esses achados fortalecem a ideia que as iniciativas públicas existentes em Belo Horizonte ainda não estão sendo eficazes, necessitando de ações mais resolutivas (FIGUEIRA; LOPES; MODENA, 2016). Assim, ressaltase que, de maneira semelhante como acontece com a de AF, o consumo alimentar também perpassa por questões além das individuais, destacando as socioculturais, econômicas e ambientais.

As modificações nos comportamentos alimentares e na AF podem explicar os resultados positivos identificados nos dados do peso corporal (MENDONÇA et al., 2015). Os resultados sugerem que o programa VAMOS foi efetivo para auxiliar na conscientização e compreensão acerca dos hábitos de atividade física e alimentares dos participantes, estimulando o aumento do gasto energético e a diminuição da ingestão calórica, o que refletiu nas diferenças de peso corporal relatadas. Corroborando com esses dados um estudo de intervenção nutricional conduzido com usuários do PAS identificou uma redução de peso de 1,3 kg entre os participantes da intervenção (MENDONÇA et al., 2015). Tais resultados são positivos visto que a redução de peso está associada ao menor risco de comorbidades relacionadas a obesidade e ao sobrepeso (RUEDA-CLAUSEN; OGUNLEYE; SHARMA, 2015). No entanto, é relevante considerar a elevada prevalência com excesso de peso (60\%) dos participantes do VAMOS (MEURER et al., 2019), o que pode estar vinculado às dificuldades de associar as intervenções de promoção da saúde com as ações de políticas públicas de acesso à alimentos mais saudáveis, conforme já apontado por Figueira, Lopes e Modena (2016).

Diante dos resultados, compreende-se que intervenções que têm como base a combinação de AF e AS favorecem a redução de peso, podendo também repercutir melhoria da percepção da QV (ARREBOLA et al., 2011). Observa-se que a QV apresenta uma relação direta e positiva com a AF e a AS. Ao considerar que ambas podem ser fatores de proteção de doenças crônicas, elas se tornam importantes para a manutenção de hábitos saudáveis (MACIEL et al., 2019; FERREIRA; MEIRELES; FERREIRA, 2018; FERREIRA; DIETTRICH; PEDRO, 2015; CAMPOS et al, 2014). Ademais, níveis satisfatórios de AF e AS são descritos em outros estudos como um diferencial na percepção da QV, especialmente por contribuir diretamente na capacidade funcional, aspectos físicos, dor e saúde geral (FEITOSA et al., 2016; FERREIRA; DIETTRICH; PEDRO, 2015; GONÇALVES et al., 2019). De acordo com os participantes, o VAMOS proporcionou mudanças em outros aspectos, como, por exemplo, na autoestima e socialização dos participantes. Essas mudanças podem estar vinculadas ao formato dos encontros (dialogado) - rodas de conversas, contribuindo, possivelmente, para a melhora da QV. Uma avaliação realizada em unidades similares a do PAS mostrou que a interação social está relacionada a uma melhora na percepção da QV de participantes de programas comunitários (FEITOSA et al., 2016).

Sendo assim, o fato dos participantes associarem o programa à uma percepção positiva em relação a adoção desses novos hábitos mais saudáveis, reforça que a implementação desse tipo programa é importante por atender o objetivo de promoção da saúde. A satisfação dos usuários é fundamental para a qualidade 
desta implantação, visto que nos serviços públicos de saúde a qualidade é primordial devido as desigualdades sociais e de acesso dos usuários, às condições de vida e saúde, a distribuição dos recursos, os custos e a ineficiência (FEITOSA et al., 2016).

O encontro dialogado e participativo, conforme proposto no VAMOS, é uma possibilidade de aproximação entre os participantes e facilita a contextualização da intervenção às características do território. Assim, caracteriza-se como oportunidade de estabelecer vínculos de amizade e confiança nas relações entre a comunidade e desta com a equipe de saúde. Estes aspectos são relevantes para ampliar o capital social, associado com a autopercepção positiva da saúde (LOCH et al., 2015; MURAYAMA; FUJIWARA; KAWACHI, 2012).

Observou-se que a intervenção foi positiva para reflexão e orientação para tomada de decisões com relação às mudanças na AF e alimentação e, na percepção dos participantes, a presença e a atitude do profissional mediador (multiplicador) dos encontros contribuíram para estas mudanças. Kardakis et al., (2013) afirmam que os profissionais de saúde desempenham um papel chave no processo de implantação de intervenções de promoção da saúde. Há evidências de associação entre a capacitação dos profissionais com a qualidade dos serviços e cuidados em saúde para a população (OLIVEIRA; WHACS, 2018; OLIVEIRA et al., 2016).

O protocolo do programa prevê o treinamento de profissionais de saúde de nível superior para conduzir os encontros e mediar as falas referentes aos temas trabalhados (JOSE et al., 2019). Este treinamento capacita os profissionais para instigar a reflexão e a autoavaliação dos participantes sobre seus comportamentos, buscando auxiliálos a identificar as possibilidades de mudanças dentro de sua realidade e motivá-los para realizar mudanças necessárias e possíveis. Galliano et al. (2016) destacam que a capacitação dos profissionais de saúde permite ampliar o leque de atendimento às necessidades da comunidade, além de facilitar a avaliação dos programas de intervenção, ampliando as chances de sucesso das estratégias adotadas.

Dentre as estratégias propostas pelo VAMOS destaca-se o desenvolvimento da autonomia, facilitando a escolha de comportamentos mais ativos e saudáveis (FRAGA et al., 2013; CAVALCANTI; OLIVEIRA, 2012). A autonomia para o autocuidado deve ser o foco das intervenções de promoção da saúde, visto que profissionais que apoiam a autonomia do participante auxiliam no processo de identificação e reflexão das dificuldades pessoais e comunitárias perante a possibilidade de mudanças de comportamento (BRASIL, 2014). No entanto, compreende-se que as escolhas são influenciadas pelos determinantes sociais. Assim, ações de intervenção para a promoção da saúde podem ter maior potencialidade se tiverem enfoque intersetorial.

Adotar um estilo de vida ativo e saudável não deve ser visto como uma ação simples de querer ou não mudar determinado comportamento. A adoção destes comportamentos é mais ampla e envolve o acesso a locais, disponibilidade de tempo, recursos apropriados e a motivação entre a oferta de AF e alimentos, além do interesse pessoal, demonstrando assim ser um processo complexo (CARVALHO; NOGUEIRA, 2016). Diante disto, as ações de promoção da saúde devem incentivar a autonomia, o empoderamento e a participação social, sem desconsiderar o entorno em que os indivíduos vivem. 
Conforme exposto por Feitosa et al. (2016) é necessário desenvolver o sentimento de pertencimento da ação. Pois, é por meio dessa percepção que os indivíduos terão acesso ao direito de realizar AF e solicitar mais espaços físicos. A população deve participar mais das decisões políticas e auxiliar na manutenção e gestão das ações propostas, lembrando que o espaço público é de todos.

O VAMOS, desde a sua criação, propõe estratégias para auxiliar as pessoas no processo de mudança de comportamento para um estilo de vida ativo e saudável. Seguindo o modelo lógico do programa, este estudo mostrou a efetividade da intervenção em curto e médio prazo por meio das mudanças relatadas pelos participantes sobre os principais marcadores do programa. No entanto, algumas limitações merecem ser observadas - apesar da contínua tentativa de considerar a realidade dos participantes do VAMOS para adaptar as estratégias de mudança de comportamento, a intervenção tem pouco poder sobre alterações no contexto ambiental. Ele também sofre limitações em suas ações quanto a articulação com outros profissionais e projetos de promoção da saúde, pois isto vai depender do vinculo interprofissional e intersetorial definidos pelos locais onde o programa é implantado. Ademais, o fato da intervenção ter sido conduzida pela pesquisadora limita as interpretações dos resultados.

\section{CONSIDERAÇÕES FINAIS}

O VAMOS propõe o uso de estratégias para auxiliar os participantes a adotarem e manterem comportamentos saudáveis de AF e alimentação. Concluiuse que na percepção dos participantes do VAMOS houve benefícios sobre os diferentes marcadores de efetividade do programa - AF, AS, QV e peso corporal. Ao que parece, a metodologia utilizada foi efetiva para atingir tais resultados. Assim, consideramos que o VAMOS se apresenta como uma possível tecnologia inovadora para ser inserida na saúde pública, especialmente se associada à outras políticas de promoção da saúde, como o PAS.

Reflexões sobre como o VAMOS poderia se articular melhor à outras ações e políticas no cenário do SUS são essenciais para que realmente esteja alinhado aos princípios da promoção da saúde. Assim, sugere-se que políticas públicas de AF e AS conversem entre si e com outros setores como de planejamento urbano, educação, segurança pública, dentre outras, a fim de estimular o trabalho intersetorial, auxiliando na superação das desigualdades de acesso à saúde.

\section{REFERÊNCIAS}

ALMEIDA, Fábio Araújo; BRITO, Fabiana Almeida; ESTABROOKS, Paul Andrew. Modelo RE-AIM: tradução e adaptação cultural para o Brasil. Família, Ciclos de Vida e Saúde no Contexto Social, v. 1, n. 1, p. 6-16, 2013.

ARAÚJO, Melissa Luciana. Repercussão da segurança alimentar e nutricional sobre o consumo de frutas e hortaliças. 2016. 126f. Dissertação (Mestrado) - Programa de PósGraduação em Enfermagem, UFMG, Belo Horizonte, 2016. 
ARREBOLA, Eloy Rodríguez et al. Evaluation of a Lifestyle Modification Program for Treatment of Overweight and Nonmorbid Obesity in Primary Healthcare and Its Influence on Health-Related Quality of Life. Nutrition in Clinical Practice, v. 26, n. 3, p. 316-321, 2011.

BANDURA, Albert. Social foundations of thought and action: a social cognitive theory. Englenwood Cliffs: Prentice Hall, 1986.

BARDIN, Laurence. Análise de conteúdo. Lisboa: Edições 70, 2009.

BECKER, Leonardo; GONÇALVES, Priscila; REIS, Rodrigo. Programas de promoção da atividade física no Sistema Único de Saúde brasileiro: revisão sistemática. Revista Brasileira de Atividade Física \& Saúde, v. 21, n. 2, p. 110-122, 2016.

BENEDETTI, Tânia Rosane Bertoldo et al. Programa "VAMOS" (Vida Ativa Melhorando a Saúde): da concepção aos primeiros resultados. Revista Brasileira de Cineantropometria e Desempenho Humano, v. 14, n. 6, p. 723-737, 2012.

BENEDETTI, Tânia Rosane Bertoldo et al. Logical model of a behavior change program for community intervention - Active Life Improving Health - VAMOS. Revista Brasileira de Atividade Física e Saúde, v. 22, n. 3, p. 309-313, 2017.

BRASIL. Ministério da Saúde. Secretaria de Vigilância Sanitária em Saúde. Departamento de Análise de Situação em Saúde. Avaliação de efetividade de programas de educação física no Brasil [recurso eletrônico]. Brasília, DF, 2013. p. 182.

BRASIL. Ministério da Saúde. Secretaria de Atenção à Saúde. Departamento de Atenção Básica. Guia alimentar para a população brasileira. 2. ed. Brasília, DF, 2014.

BRASIL. Portaria $n^{\circ} 2.436$, de 21 de setembro de 2017. Aprova a Política Nacional de Atenção Básica, estabelecendo a revisão de diretrizes para a organização da Atenção Básica, no âmbito do Sistema Único de Saúde (SUS). Diário Oficial da União: seção 1, Brasília, DF, n. 183, p. 68, 22 set. 2017.

CAMPOS, Ana Cristina Viana, et al. Qualidade de vida de idosos praticantes de atividade física no contexto da estratégia saúde da família. Texto e Contexto Enfermagem, v. 23, n. 4, p. 889-897, 2014.

CAMPOS, Leonardo de. Análise de custo de intervenção para a promoção de atividade física em Unidades de Saúde da Família de Rio Claro - SP. 2017. 102 f. Dissertação (Mestrado) - Programa de Pós-graduação em Ciências da Motricidade. Universidade Estadual Paulista, Rio Claro, SP. 2017.

CARVALHO, Fabio Fortunato Brasil; NOGUEIRA, Julia Aparecida Devidé. Práticas corporais e atividades físicas na perspectiva da Promoção da Saúde na Atenção Básica. Ciência \& Saúde Coletiva, v. 21, n. 6, p. 1829-1838, 2016.

CAVALCANTI, Ana Maria; OLIVEIRA, Angela Cristina Lucas de (ORG). Autocuidado apoiado: manual do profissional de saúde. Curitiba: Secretaria Municipal da Saúde, 2012.

FEITOSA, Wallacy Milton do Nascimento. User's perception of actions, improvement in quality of life and satisfaction whit the Academia da Cidade Program. Revista Brasileira de Atividade Física e Saúde, v. 21, n. 5, p. 461-469, 2016.

FERREIRA, Joel Saraiva; DIETTRICH, Sandra Helena Correia; PEDRO, Danielly Amado. Influência da prática de atividade física sobre a qualidade de vida de usuários do SUS.

Saúde Debate, v. 39, n. 106, p. 792-801, 2015. 
FERREIRA, Luana Karoline; MEIRELES Juliana Fernandes Filgueiras; FERREIRA, Maria Elisa Caputo. Avaliação do estilo e qualidade de vida em idosos: uma revisão de literatura. Revista Brasileira de Geriatria e Gerontologia, v. 21, n. 5, p. 639-651, 2018.

FIGUEIRA, Taís Rocha; LOPES, Aline Cristine Souza; MODENA, Celina Maria. Barreiras e fatores promotores do consumo de frutas e hortaliças entre usuários do Programa Academia da Saúde. Revista de Nutrição, v. 29, n. 1, p. 85-95, 2016.

FLORINDO, Alex Antonio, et al. Physical activity promotion in primary health care in Brazil: a counseling model applied to community health workers. Journal of Physical Activity and Health, v. 11, n. 8, p.1531-1539. 2014.

FLORINDO, Alex Antonio et al. Description of health promotion actions in Brazilian cities that received funds to develop "Academia da Saúde" Program. Revista Brasileira de Cineantropometria e Desempenho Humano, v. 18, n. 4, p. 483-492. 2016.

FRAGA, Alex Branco et al. Curso de extensão em Promoção de Saúde para gestores do SUS com enfoque no Programa Academia da Saúde. Brasília,DF: Ministério da Saúde, 2013.

GALLIANO, Leony et al. Intervenção com atividade física em uma Unidade Básica de Saúde - Projeto UBS+ativa: aspectos metodológicos. Revista Brasileira de Atividade Física e Saúde, v. 21, n. 6, p. 571-580, 2016.

GERAGE, Aline Mendes et al. Effectiveness of a behavior change program on physical activity and eating habits in patients with hypertension: a randomized controlled trial. Journal of Physical Activity and Health, v. 14, n. 12, p. 943 - 952, 2017.

GLASGOW, Russel E.; VOGT, Thomas M.; BOLES, Shaw M. Evaluating the public health impact of health promotion interventions: the RE-AIM framework. American Journal of Public Health, v. 89, n. 9, p. 1322 - 1327, 1999.

GONÇALVES, Laís Barreto de Brito et al. Programa academia da saúde: operacionalidade, ações e integração. Revista Brasileira em Promoção da Saúde, v. 32, p. 1-11, 2019.

JOSÉ, Helma Pio Mororó, et al. Validação do treinamento on-line para multiplicadores do programa vida ativa melhorando a saúde (VAMOS). Journal of Physical Education, v. 30, e3040, p. 1-11, 2019.

KARDAKIS, Therese, et al. Lifestyle interventions in primary health care: professional and organizational challenges. European Journal of Public Health, v. 24, n. 1, p. 79 - 84, 2013.

KNUTH, Alan Goularte et al. Avaliação de programas de atividade física no Brasil: uma revisão de evidências em experiências selecionadas. In: BRASIL. Ministério da Saúde. Secretaria de Vigilância em Saúde. Departamento de Análise de Situação em Saúde. Saúde Brasil 2010: uma análise da situação de saúde e de evidências selecionadas de impacto de ações de vigilância em saúde. Brasília, 2011. p. 349-360.

KONRAD, Lisandra. Maria et al. Avaliação de programas de mudança de comportamento usando a ferramenta RE-AIM: um estudo de revisão sistemática. Revista Brasileira de Atividade Física \& Saúde, v. 22, n. 5, p. 448 - 458, 2018.

$\mathrm{LOCH}$, Mathias Roberto, et al. Association between social capital and self-perception of health in Brazilian adults. Revista de Saúde Pública, v. 49, n. 53, p. 1 - 9, 2015.

LOPES, Aline Cristine Souza; MENEZES, Mariana Carvalho; ARAÚJO, Melissa Luciana. O ambiente alimentar e o acesso a frutas e hortaliças: "uma metrópole em perspectiva". Saúde e Sociedade, v. 26, n. 3, p. 764-773, 2017. 
MACIEL, Marcos Gonçalves, et al. Avaliação de desempenho do Programa Academia da Saúde em Belo Horizonte: um estudo de caso. Movimento, v. 25, e25026, 2019.

MALTA, Deborah et al. Política Nacional de Promoção da Saúde, descrição da implementação do eixo atividades físicas e práticas corporais, 2006 a 2014. Revista Brasileira de Atividade Física \& Saúde, v. 19, n. 3, p. 286-299, 2014.

MENDONÇA, Raquel de Deus et al. The impact of a nutritional intervention on the nutritional status and anthropometric profile of participants in the health Gym Programme in Brazil.

Ciência e Saúde Coletiva, v. 20, n. 6, p. 1937-1946, 2015.

MEURER, Simone Teresinha. Avaliação da estratégia de promoção de atividade física e alimentação saudável - VAMOS - no programa academia da saúde de Belo Horizonte, Minas Gerais. 2016. 184 f. Tese (Doutorado) - Programa de Pós-Graduação em Educação Física. UFSC, Florianópolis, 2016.

MEURER, Simone Teresinha et al. Effectiveness of the VAMOS Strategy for Increasing Physical Activity and Healthy Dietary Habits: A Randomized Controlled Community Trial. Health Education e Behavior, v. 46, p. 406-416, 2019.

MINAYO, Maria Cecília de Souza. O desafio do conhecimento: pesquisa qualitativa em saúde. São Paulo/Rio de Janeiro: Hucitec/Abrasco, 1992.

MURAYAMA, Hiroshi; FUJIWARA, Yoshinori; KAWACHI, Ichiro. Social capital and health: a review of prospective multilevel studies. Journal of Epidemiology, v. 22, n. 3, p. 179-187, 2012.

OLIVEIRA, Braulino Nogueira de; WACHS, Felipe. Educação Física e Atenção Primária à Saúde: apropriações acerca do apoio matricial. Movimento, v. 24, n.1, p. 173-186, 2018.

OLIVEIRA, Cristiano de Souza; GORDIA, Alex Pinheiro; QUADROS, Teresa Maria Bianchini. Mudanças na atividade física de universitários: associação com informações sobre saúde e acesso a locais para prática. Revista de Salud Pública, v. 19, n. 5, p. 617-623, 2017.

OLIVEIRA, Mariana Policena Rosa de et al. Formação e qualificação de profissionais de saúde: fatores associados à qualidade da atenção primária. Revista Brasileira de Educação Médica, v. 40, n. 4, p. 547-559, 2016.

RIBEIRO, Evelyn Helena Corgosinho et al. Avaliação da efetividade de intervenções de promoção da atividade física no Sistema Único de Saúde. Revista de Saúde Pública, v. 51, n.1, p.51-56, 2017.

RUEDA-CLAUSEN, Christian F.; OGUNLEYE, Ayodele A.; SHARMA, Arya Mitra. Health Benefits of Long-Term Weight-Loss Maintenance. Annual Review of Nutrition, v. 35, p. 475-516, 2015.

SÁ, Gisele Balbino Araújo Rodrigues de et al. O Programa Academia da Saúde como estratégia de promoção da saúde e modos de vida saudáveis: cenário nacional de implementação. Ciência e Saúde Coletiva, v. 21, n. 6, p. 1849-1859, 2016.

TONG, Alisson; SAINSBURY, Peter, CRAIG, Jonathan. Consolidated criteria for reporting qualitative research (COREQ): a 32-item checklist for interviews and focus groups. International Journal for Quality in Health Care, v. 19, n. 6, p. 349 - 357, 2007.

TONOSAKI, Lucia Midori Damaceno et al. Barreiras e facilitadores para a participação em um programa de mudança de comportamento: análise de grupos focais. Revista Brasileira de Ciências do Esporte, v. 40, n.2, p.138-145, 2018. 
WORLD HEALTH ORGANIZATION. Plano de Ação Global para a Atividade Física 20182030: versão síntese Mais Pessoas Ativas para um Mundo Mais Saudável. Geneva, 2018.

\section{Financiamento:}

"Programa de Pesquisa para o SUS" (PPSUS / FAPESC / 2014 / TR / 2263) e Conselho Nacional de Desenvolvimento Científico e Tecnológico (CNPq n.14 / 2012 AN 475075 / 2012-9). 